\title{
On the Structural Stability of Mutualistic Systems
}

\author{
Rudolf P. Rohr ${ }^{1,2 *}$, Serguei Saavedra ${ }^{1 *}$, and Jordi Bascompte ${ }^{1 \dagger}$ \\ ${ }^{1}$ Integrative Ecology Group \\ Estación Biológica de Doñana (EBD-CSIC) \\ Calle Américo Vespucio s/n \\ E-41092 Sevilla, Spain \\ ${ }^{2}$ Unit of Ecology and Evolution \\ Department of Biology, University of Fribourg \\ Chemin du Musée 10 \\ CH-1700 Fribourg, Switzerland
}

One sentence summary: We study the range of perturbations biodiversity can withstand and how this is modulated by the network of interspecific interactions.

*These authors contributed equally to this work

${ }^{\dagger}$ To whom correspondence should be addressed. E-mail: bascompte@ebd.csic.es 
In theoretical ecology, traditional studies based on dynamical stability and numerical simulations have not found a unified answer to the effect of network architecture on community persistence. Here, we introduce a mathematical framework based on the concept of structural stability to explain such a disparity of results. We investigate the range of conditions necessary for the stable coexistence of all species in mutualistic systems. We show that the apparently contradictory conclusions reached by previous studies arise as a consequence of overseeing either the necessary conditions for persistence or its dependence on model parameterization. We show that observed network architectures maximize the range of conditions for species coexistence. We discuss the applicability of structural stability to study other types of interspecific interactions.

A prevailing question in ecology (particularly since May's (1) seminal work in the early 1970s) is whether, given an observed number of species and their interactions, there are ways to organize those interactions that lead to more persistent communities. Conventionally, studies addressing this question have either looked into local stability or used numerical simulations (2-4). However, these studies have not found a unified answer yet $(1,5-12)$. Therefore, the current challenge is to develop a general framework to provide a unified assessment of the implications of the architectural patterns of the networks we observe in nature.

\section{Main approaches in theoretical ecology}

\section{Dynamical stability and feasibility}

Studies based on the mathematical notions of local stability, D-stability, and global stability have advanced our knowledge on what makes ecological communities stable. In 
1 particular, these studies explore how interaction strengths need to be distributed across 2 species so that an assumed feasible equilibrium point can be stable $(1-4,13-17)$. By definition, a feasible equilibrium point is that in which all species have a constant positive abundance across time. Note that a negative abundance makes no sense biologically and an abundance of zero would correspond to an extinct species.

The dynamical stability of a feasible equilibrium point corresponds to the conditions under which the system returns to the equilibrium point after a perturbation in species abundance. Local stability, for instance, looks at whether a system will return to an assumed feasible equilibrium after an infinitesimal small perturbation (1-3,13). D-stability, in turn, looks at the local stability of any potential feasible equilibrium that the system may have (15-17). More generally, global stability looks at the stability of any potential feasible equilibrium point after a perturbation of any given amplitude (14-17). Note 18 provides a technical definition of these different types of dynamical stability and their relationship.

In most of these stability studies, however, a feasible equilibrium point is always assumed without rigorously studying the set of conditions allowing its existence $(5,14,15$, 19). Yet, in any given system, we can find examples where we satisfy only one, both, or none of the feasibility and stability conditions $(3,16,17,19)$. This means that without a proper consideration of the feasibility conditions, any conclusion for studying the stable coexistence of species is based on a system that may or may not exist $(3,5,19)$.

To illustrate this point, consider the following textbook example of a two-species competition system:

$$
\left\{\begin{array}{c}
\frac{d N_{1}}{d t}=N_{1}\left(\alpha_{1}-\beta_{11} N_{1}-\beta_{12} N_{2}\right) \\
\frac{d N_{2}}{d t}=N_{2}\left(\alpha_{2}-\beta_{21} N_{1}-\beta_{22} N_{2}\right)
\end{array},\right.
$$

where $N_{1}$ and $N_{2}$ are the abundances of species 1 and $2 ; \beta_{11}$ and $\beta_{22}$ are their intraspecific competition strengths; $\beta_{12}$ and $\beta_{21}$ are their interspecific competition strengths; and $\alpha_{1}$ and $\alpha_{2}$ are their intrinsic growth rates. An equilibrium point of the system is a pair of 
1 abundances $N_{1}^{*}$ and $N_{2}^{*}$ that makes the right side of the ordinary differential equation 2 system equal to zero.

3

4

5

${ }_{6} \frac{\beta_{22} \alpha_{1}-\beta_{12} \alpha_{3}}{\beta_{11} \beta_{22}-\beta_{12} \beta_{21}}>0$ and $N_{2}^{*}=\frac{\beta_{11} \alpha_{2}-\beta_{21} \alpha_{1}}{\beta_{11} \beta_{22}-\beta_{12} \beta_{21}}>0(3,4,19)$. This implies that if we set, for

7 example, $\beta_{11}=\beta_{22}=1, \beta_{12}=\beta_{21}=0.5, \alpha_{1}=1$, and $\alpha_{2}=2$ we fulfil the stability

While the only condition necessary to guarantee the global stability of any feasible equilibrium point in this system is that the interspecific competition strengths are lower than the intraspecific ones $\left(\beta_{12} \beta_{21}<\beta_{11} \beta_{22}\right)$, the feasibility conditions are given by $N_{1}^{*}=$ condition but not the feasibility condition, while if we set $\beta_{11}=\beta_{22}=0.5, \beta_{12}=\beta_{21}=1$, $\alpha_{1}=\alpha_{2}=1$ we can satisfy the feasibility condition but not the stability one. To have a stable and feasible equilibrium point we need to set, for instance, $\beta_{11}=\beta_{22}=1$, $\beta_{12}=\beta_{21}=0.5$, and $\alpha_{1}=\alpha_{2}=1$ (see Fig. 1 for a graphical illustration).

The example above confirms the importance of verifying both the stability and the feasibility conditions of the equilibrium point when analyzing the stable coexistence of species $(3-5,19)$. Of course, we can always fine tune the parameter values of intrinsic growth rates such that the system is feasible $(16,17)$. This strategy, for example, has been used when studying the success probability of an invasive species (20). However, when fixing the parameter values of intrinsic growth rates, we are not any more studying the overall effect of interspecific interactions on the stable coexistence of species. Rather, we are answering the question of how interspecific interactions increase the persistence of species for a given parameterization of intrinsic growth rates. As we will show below, this is also the core of the problem in studies based on arbitrary numerical simulations.

\section{Numerical simulations}

Numerical simulations have provided an alternative and useful tool to explore species coexistence in large ecological systems where analytical solutions are precluded (3). Under this approach, one has as a prerequisite to parameterize the dynamical model, or a least to have a good estimate of the statistical distribution from which these parameters should 
1 be sampled. However, if one chooses an arbitrary parameterization without an empirical 2 justification, any study has a high chance of being inconclusive for real ecosystems since 3 species persistence is strongly dependent on the chosen parameterization.

To illustrate this point, let us simulate the dynamics of an ecological model (6) with three different parameterizations of intrinsic growth rates (21). Additionally, these simulations are performed over an observed mutualistic network of interactions between flowering plants and their pollinators located in Hickling Norfolk, UK (see Table S1), a randomized version of this observed network, and the observed network without mutualistic interactions (i.e., we assume that there is only competition among plants and among animals). Figure 2 shows that it is possible to find a set of intrinsic growth rates such that any network that we analyze is completely persistent and, at the same time, the alternative networks are less persistent.

This observation has two important implications. First, this means that by using different parameterizations for the same dynamical model and network of interactions, one can observe from all to a few of the species surviving. Second, this means that each network has a limited range of parameter values under which all species coexist. Thus, by studying a specific parameterization, for instance, one could wrongly conclude that a random network has a higher effect on community persistence than an observed network, or vice versa (10-12). This sensitivity to parameter values clearly illustrates that the conclusions that arise from studies using arbitrary values in intrinsic growth rates are not about the effects of network architecture of species coexistence, but about which network architecture maximizes species persistence for that specific parameterization.

The above remarks reveal that traditional studies focusing on either local stability or numerical simulations can lead to apparently contradictory results. Therefore, we need a different conceptual framework to unify results and seek for appropriate generalizations. 


\section{Structural stability}

2 Structural stability has been a general mathematical approach to study the behavior of dynamical systems. A system is considered to be structurally stable if any smooth change in the model itself or in the value of its parameters does not change its dynamical behavior 5 (e.g., the existence of equilibrium points, limit cycles, or deterministic chaos) (22-25). In 6 the context of ecology, an interesting behavior is the stable coexistence of species, i.e., the 7 existence of an equilibrium point that is feasible and dynamically stable. For instance, in our previous two-species competition system, there is a restricted area in the parameter space of intrinsic growth rates that leads to a globally stable and feasible solution as long as $\rho<1$ (white area in Fig. 3). Importantly, Figure 3 also reveals that the higher the competition strength $\rho$, the larger the size of this restricted area $(19,26)$. Therefore, a relevant question here is not only whether the system is structurally stable or not, but how large is the domain in the parameter space leading to the stable coexistence of species.

To address the above question, here we recast the mathematical definition of structural stability to that in which a system is more structurally stable, the larger the area of parameter values leading to both a dynamically stable and feasible equilibrium (27-29). This means that a highly structurally stable ecological system is more likely to be stable and feasible by handling a wider range of conditions before the first species becomes extinct. Previous studies have used this approach in low-dimensional ecological systems $(3,19)$. Yet, due to its complexity, almost no study has fully developed this rigorous analysis for a system with an arbitrary number of species. A significant exception has been the use of structural stability to calculate an upper bound to the number of species that can coexist in a given community $(6,30)$.

Here, we introduce this extended concept of structural stability into community ecology to study the extent to which network architecture - strength and organization of interspecific interactions - modulates the range of conditions compatible with the stable coexistence of species. As an empirical application of our framework, we study the 
1 structural stability of mutualistic systems and apply it on a dataset of 23 quantitative mutualistic networks (Table S1). We surmise that observed network architectures increase the structural stability and in turn the likelihood of species coexistence as function of the possible set of conditions in an ecological system. We discuss the applicability of our framework to other types of interspecific interactions in complex ecological systems.

\section{- Structural stability of mutualistic systems}

Mutualistic networks are formed by the mutually-beneficial interactions between flowering plants and their pollinators or seed dispersers (31). Importantly, these mutualistic networks have been shown to share a nested architectural pattern (32). This nested architecture means that, typically, the mutualistic interactions of specialist species are proper subsets of the interactions of more generalist species (32). While it has been repeatedly shown that this nested architecture may arise from a combination of life-history and complementarity constraints among species (32-35), the effect of this nested architecture on community persistence continues to be a matter of strong debate. On the one hand, it has been shown that a nested architecture can facilitate the maintenance of species coexistence (6), exhibit a flexible response to environmental disturbances $(7,8,36)$, and maximize total abundance (12). On the other hand, it has also been suggested that this nested architecture can minimize local stability (9), have a negative effect on community persistence (10), and have a low resilience to perturbations (12). Not surprisingly, the majority of these studies have been based on either local stability or numerical simulations with arbitrary parameterizations (but see Ref. 6).

\section{Model of mutualism}

To study the structural stability and explain the apparently contradictory results found in studies of mutualistic networks, we first need to introduce an appropriate model describing the dynamics between and within plants and animals. We use the same set of differential 
1 equations as in Ref. 6. We choose these dynamics because they are simple enough to 2 provide analytical insights, and yet complex enough to incorporate key elements - such 3 as saturating, functional responses $(37,38)$ and interspecific competition within a guild 4 (6) - recently adduced as necessary ingredients for a reasonable theoretical exploration of 5 mutualistic interactions. Specifically, the dynamical model has the following form:

$$
\left\{\begin{array}{c}
\frac{d P_{i}}{d t}=P_{i}\left(\alpha_{i}^{(P)}-\sum_{j} \beta_{i j}^{(P)} P_{j}+\frac{\sum_{j} \gamma_{i j}^{(P)} A_{j}}{1+h \sum_{j} \gamma_{i j}^{(P)} A_{j}}\right) \\
\frac{d A_{i}}{d t}=A_{i}\left(\alpha_{i}^{(A)}-\sum_{j} \beta_{i j}^{(A)} A_{j}+\frac{\sum_{j} \gamma_{i j}^{(A)} P_{j}}{1+h \sum_{j} \gamma_{i j}^{(A)} P_{j}}\right)
\end{array},\right.
$$

where the variables $P_{i}$ and $A_{i}$ denote the abundance of plant and animal species $i$, respectively. The parameters of this mutualistic system correspond to the values describing intrinsic growth rates $\left(\alpha_{i}\right)$, intra-guild competition $\left(\beta_{i j}\right)$, the benefit received via mutualistic interactions $\left(\gamma_{i j}\right)$, and the saturating constant of the beneficial effect of mutualism $(h)$, commonly known as the handling time. Since our main focus is on mutualistic interactions, we keep as simple as possible the competitive interactions for the sake of analytical tractability. In the absence of empirical information about interspecific competition, we use a mean field approximation for the competition parameters (6), where we $\operatorname{set} \beta_{i i}^{(P)}=\beta_{i i}^{(A)}=1$ and $\beta_{i j}^{(P)}=\beta_{i j}^{(A)}=\rho<1(i \neq j)$.

Following Ref. 39, the mutualistic benefit can be further disentangled by $\gamma_{i j}=$ $\left(\gamma_{0} y_{i j}\right) /\left(k_{i}^{\delta}\right)$, where $y_{i j}=1$ if species $i$ and $j$ interact and zero otherwise; $k_{i}$ is the number of interactions of species $i$; $\gamma_{0}$ represents the level of mutualistic strength, and $\delta$ corresponds to the mutualistic trade-off. Recall that the mutualistic strength is the per-capita effect of a certain species on the per-capita growth rate of their mutualistic partners. The mutualistic trade-off modulates the extent to which a species that interacts with few other species does it strongly, while a species that interacts with many partners does it weakly. This trade-off has been justified on empirical grounds $(40,41)$. Importantly, the degree to which interspecific interactions $y_{i j}$ are organized into a nested way can be quantified by the value of nestedness $N$ introduced in Ref. 42 . 
We are interested in quantifying the extent to which network architecture (i.e., the combination of mutualistic strength, mutualistic trade-off, and nestedness) modulates the set of conditions compatible with the stable coexistence of all species, i.e., the structural stability. In the next sections, we explain how this problem can be split into two parts. First, we explain how the stability conditions can be disentangled from the feasibility conditions, as it has already been shown for the two-species competition system. Specifically, we show that below a critical level of mutualistic strength $\left(\gamma_{0}<\gamma_{0}^{r}\right)$, any feasible equilibrium point is granted to be globally stable. Second, we explain how network architecture modulates the domain in the parameter space of intrinsic growth rates leading to a feasible equilibrium under the constraints of being globally stable (given by the level of mutualistic strength).

\section{Stability condition}

In this section, we investigate the conditions in our dynamical system that any feasible equilibrium point needs to satisfy to be globally stable. To derive these conditions, we start by studying the linear Lotka-Volterra approximation (i.e., $h=0$ ) of the dynamical model (Equation 2). In this linear approximation, the model reads

$$
\left[\begin{array}{l}
\frac{d P}{d t} \\
\frac{d A}{d t}
\end{array}\right]=\operatorname{diag}\left(\left[\begin{array}{l}
P \\
A
\end{array}\right]\right)(\left[\begin{array}{l}
\alpha^{(P)} \\
\alpha^{(A)}
\end{array}\right]-\underbrace{\left[\begin{array}{cc}
\beta^{(P)} & -\gamma^{(P)} \\
-\gamma^{(A)} & \beta^{(A)}
\end{array}\right]}_{:=B}\left[\begin{array}{l}
P \\
A
\end{array}\right]),
$$

where the matrix $B$ is a two-by-two block matrix embedding all the interaction strengths.

Conveniently, the global stability of a feasible equilibrium point in this linear LotkaVolterra model has already been studied $(14-17,43)$. Particularly relevant in here is remembering that an interaction matrix that is Lyapunov-diagonally stable grants the global stability of any potential feasible equilibrium (14-18).

While it is mathematically difficult to verify the condition for Lyapunov-diagonal stability, it is known that for some classes of matrices, Lyapunov stability and Lyapunovdiagonal stability are equivalent conditions (44). Importantly, symmetric matrices and 
$1 Z$-matrices (i.e., matrices whose off-diagonal elements are non-positive) belong to those 2 classes of equivalent matrices. Note that our interaction strength matrix $B$ is either symmetric when the mutualistic trade-off is zero $(\delta=0)$ or a $Z$-matrix when the interspecific competition is zero $(\rho=0)$. This means that as long as the real parts of all eigenvalues of $B$ are positive (18), any feasible equilibrium point is globally stable. For instance, in the case of $\rho<1$ and $\gamma_{0}=0$, the interaction matrix $B$ is symmetric and Lyapunov-diagonally stable since its eigenvalues are $1-\rho,\left(S_{A}-1\right) \rho+1$, and $\left(S_{P}-1\right) \rho+1$.

$8 \quad$ Note that for $\rho>0$ and $\delta>0$, there are no analytical results yet demonstrating that 9 Lyapunov-diagonal stability is equivalent to Lyapunov stability. However, after intensive numerical simulations, we conjecture that the two main consequences of Lyapunovdiagonal stability hold (45). Specifically, we state the following conjectures:

Conjecture 1: if $B$ is Lyapunov stable, then $B$ is $D$-stable.

Conjecture 2: if $B$ is Lyapunov stable, then any feasible equilibrium is globally stable.

Importantly, we find that for any given mutualistic trade-off and interspecific competition, the higher the level of mutualistic strength, the smaller the maximum real part of the eigenvalues of $B(45)$. This means that there is a critical value of mutualistic strength $\left(\gamma^{r}\right)$ such that above this level the matrix $B$ is not any more Lyapunov stable. To compute $\gamma_{0}^{r}$, we just need to find the critical value of $\gamma_{0}$ at which the real part of one of the eigenvalues of the interaction-strength matrix reaches zero (45). This implies that at least below this critical value $\gamma_{0}<\gamma^{r}$, any feasible equilibrium is granted to be locally and globally stable according to conjectures 1 and 2 , respectively. Note that we can also grant the global stability of matrix $B$ by the condition of being positive definite, which is even stronger than Lyapunov-diagonal stability (14). However, this condition imposes stronger constraints on the critical value of mutualistic strength than Lyapunov stability (39).

Finally, we study the stability conditions for the nonlinear Lotka-Volterra system (Equation 2). While the theory has been developed for the linear Lotka-Volterra system, 
1 we explain how it can be extended to the nonlinear dynamical system. To grant the 2 stability of any feasible equilibrium (i.e., $P_{i}>0$ and $A_{i}>0$ for all $i$ ) in the nonlinear 3 system, we need to show that the above stability conditions hold on the following two4 by-two block matrix $(14,43)$ :

$$
B_{n l}:=\left[\begin{array}{cc}
\beta_{i j}^{(P)} & -\frac{\gamma_{i j}^{(P)}}{1+h \sum_{k} \gamma_{i k}^{(P)} A_{k}} \\
-\frac{\gamma_{i j}^{(A)}}{1+h \sum_{k} \gamma_{i k}^{(A)} P_{k}} & \beta_{i j}^{(A)}
\end{array}\right] .
$$

Note that $B_{n l}$ differs from $B$ only in the off-diagonal block with a decreased mutualistic strength. This implies that the critical value of mutualistic strength for the nonlinear Lotka-Volterra system is larger or equal than the critical value for the linear system (45). Therefore, the critical value $\gamma^{r}$ derived from the linear Lotka-Volterra system (i.e., from the matrix $B$ ) is already a sufficient condition to grant the global stability of any feasible equilibrium in the nonlinear case. However, this does not imply that above this critical value of mutualistic strength a feasible equilibrium is unstable. In fact, when the mutualistic-interaction terms are saturated $(h>0)$, it is possible to have feasible and locally stable equilibria for any level of mutualistic strength $(39,45)$.

\section{Feasibility condition}

We highlight that for any interaction strength matrix $B$, whether it is stable or not, it is always possible to find a set of intrinsic growth rates such that the system is feasible (Fig. 2). To find this set of values, we just need to choose a feasible equilibrium point, such that the abundance of all species is greater than zero $\left(A_{i}^{*}>0\right.$ and $\left.P_{j}^{*}>0\right)$, and find the vector of intrinsic growth rates such that the right side of Equation 2 is equal to zero, i.e., $\alpha_{i}^{(P)}=\sum_{j} \beta_{i j}^{(P)} P_{j}^{*}-\frac{\sum_{j} \gamma_{i j}^{(P)} A_{j}^{*}}{1+h \sum_{j} \gamma_{i j}^{(P)} A_{j}^{*}}$ and $\alpha_{i}^{(A)}=\sum_{j} \beta_{i j}^{(A)} A_{j}^{*}-\frac{\sum_{j} \gamma_{i j}^{(A)} P_{j}^{*}}{1+h \sum_{j} \gamma_{i j}^{(A)} P_{j}^{*}}$. Note that this reconfirms that the stability and feasibility conditions are different and they need to be rigorously verified when studying the stable coexistence of species $(3,16,17,19)$. Importantly, this also highlights that the relevant question is not whether we can find a feasible equilibrium point, but how large is the domain of intrinsic growth rates leading 
to a feasible and stable equilibrium point. We call this domain the feasibility domain.

Since the parameter space of intrinsic growth rates is substantially large $\left(\mathbf{R}^{S}\right.$, where $S$ is the total number of species), an exhaustive numerical search of the feasibility domain is impossible. However, we can analytically estimate the center of this domain with what we call the structural vector of intrinsic growth rates. For example, in the two-species competition system of Figure 4A, the structural vector is the vector (in red), which is in the center of the domain leading to feasibility of the equilibrium point (white region). Note that any vector of intrinsic growth rates collinear to the structural vector guarantees the feasibility of the equilibrium point, i.e., guarantees species coexistence. Importantly, since the structural vector is the center of the feasibility domain then it is also the vector that can tolerate the strongest deviation before leaving the feasibility domain, i.e., before having at least one species going extinct.

In mutualistic systems, we need to find a structural vector for animals and another one for plants. Recall that these structural vectors are the set of intrinsic growth rates that allow the strongest perturbations before leaving the feasibility domain. To find these structural vectors, we have to transform the interaction-strength matrix $B$ to an effective competition framework (45). This results in an effective competition matrix for plants and a different one for animals (6), where these matrices represent respectively the apparent competition among plants and among animals once taking into account the indirect effect via their mutualistic partners. Note that with a non-zero mutualistic trade-off $(\delta>0)$, the effective competition matrices are non-symmetric, and in order to find the structural vectors we have to use the singular decomposition approach, i.e., a generalization of the eigenvalues decomposition. This results in a left and a right structural vector for plants and for animals in the effective competition framework. Finally, we need to move back from the effective competition framework to obtain a left and right vector for plants $\left(\alpha_{L}^{(P)}\right.$ and $\left.\alpha_{R}^{(P)}\right)$ and animals $\left(\alpha_{L}^{(A)}\right.$ and $\left.\alpha_{R}^{(A)}\right)$ in the observed mutualistic framework. The full derivation is provided in the SM (45). 
Once we locate the center of the feasibility domain with the structural vectors, we can approximate the boundaries of this domain by quantifying the amount of variation from the structural vectors allowed by the system before having any of the species going extinct, i.e., before losing the feasibility of the system. To quantify this amount, we introduce proportional random perturbations to the structural vectors, generate numerically the new equilibrium points (21), and measure the angle or the deviation between the structural vectors and the perturbed vectors (for a graphical example see Fig. 4A). The deviation from the structural vectors is quantified, for the plants, by $\eta_{P}\left(\alpha^{(P)}\right)=\left(1-\cos \left(\theta_{L}^{(P)}\right) \cos \left(\theta_{R}^{(P)}\right)\right) /\left(\cos \left(\theta_{L}^{(P)}\right) \cos \left(\theta_{R}^{(P)}\right)\right)$, where $\theta_{L}^{(P)}$ and $\theta_{R}^{(P)}$ are, respectively, the angles between $\alpha^{(P)}$ and $\alpha_{L}^{(P)}$ and between $\alpha^{(P)}$ and $\alpha_{R}^{(P)}$. Note that $\alpha^{(P)}$ is any perturbed vector of intrinsic growth rates of plants. The deviation from the structural vector of animals is computed similarly.

Importantly, Figure 4B shows that the larger is the deviation of the perturbed intrinsic growth rates from the structural vectors, the lower is the persistence of the community as defined by the fraction of surviving species. This confirms that there is a restricted domain of intrinsic growth rates centered on the structural vectors compatible with the stable coexistence of species. The higher the tolerated deviation from the structural vectors within which all species coexist, the higher the feasibility domain, and in turn the higher the structural stability of the system.

\section{Network architecture and structural stability}

To investigate the extent to which network architecture modulates the structural stability of mutualistic systems, we explore the combination of alternative network architectures (i.e., combinations of nestedness, mutualistic strength, and mutualistic trade-off) and their corresponding feasibility domains.

To explore these combinations, for each observed mutualistic network (see Table S1), we obtain 250 different model-generated nested architectures by using an exhaustive re- 
1 sampling model $(46)$ that preserves the number of species and the expected number of interactions (45). Theoretically, nestedness ranges from 0 to 1 (42). However, if one imposes architectural constraints such as preserving the number of species and interactions, the effective range of nestedness that the network can exhibit may be smaller (45). Additionally, each individual model-generated nested architecture is combined with different levels of mutualistic trade-off $\delta$ and mutualistic strength $\gamma_{0}$. For the mutualistic trade-off, we explore values $\delta \in[0, \ldots, 1.5]$ with steps of 0.05 that allow us to explore sub-linear, linear, and superlinear trade-offs. Note that the case $\delta=0$ is equivalent to the soft mean field approximation studied in Ref. 6. Recall that for each combination of network of interactions and mutualistic trade-off, there is a unique critical value $\gamma_{0}^{r}$ in the level of mutualism strength $\gamma_{0}$ up to which any feasible equilibrium is globally stable. This critical value $\gamma_{0}^{r}$ is dependent on the mutualistic trade-off and nestedness. However, the mean mutualistic strength $\bar{\gamma}=\left\langle\gamma_{i j}\right\rangle$ shows no pattern as function of mutualistic trade-off and nestedness (45). Therefore, we explore values of $\gamma_{0} \in\left[0, \ldots, \gamma_{0}^{r}\right]$ with steps of 0.05 and calculate the new generated mean mutualistic strengths. This produced a total of $250 \times 589$ different network architectures (nestedness, mutualistic trade-off, and mean mutualistic strength) for each observed mutualistic network.

We quantify how the structural stability (feasibility domain) is modulated by these alternative network architectures in the following way. First, we compute the structural vectors of intrinsic growth rates that grant the existence of a feasible equilibrium of each alternative network architecture. Second, we introduce proportional random perturbations to the structural vectors of intrinsic growth rates, and measure the angle or deviation between the structural vectors and the perturbed vectors $\left(\eta_{(A)}, \eta_{(P)}\right)$. Third, we simulate species abundance using the mutualistic model of Ref. 6 and the perturbed growth rates as intrinsic growth rate parameter values (21). These deviations lead to parameter domains from all to a few species surviving (Fig. 4).

Finally, we quantify the extent to which network architecture modulates structural 
1 stability by looking at the association of community persistence with network architecture

2 parameters, once taking into account the effect of intrinsic growth rates. Specifically, we study this association using the partial fitted values from a binomial regression (47) of

4 the fraction of surviving species on nestedness $(N)$, mean mutualistic strength $(\bar{\gamma})$, and 5 mutualistic trade-off $(\delta)$, while controlling for the deviations from the structural vectors 6 of intrinsic growth rates $\left(\eta_{(A)}, \eta_{(P)}\right)$. The full description of this binomial regression and 7 the calculation of partial fitted values are provided in Note 48. Note that these partial 8 fitted values are the contribution of network architecture to the logit of the probability of species persistence, and in turn, these values are positively proportional to the size of the feasibility domain.

\section{${ }_{11}$ Results}

We analyze each observed mutualistic network independently since network architecture is constrained to the properties of each mutualistic system (11). For a given pollination system located in the KwaZulu-Natal region of South Africa, Figure 5 shows the extent to which its network architecture modulates structural stability. Specifically, the figure plots the partial fitted values as a function of network architecture. Importantly, Figure 5A shows that not all architectural combinations have the same structural stability. In particular, the architectures that maximize structural stability (reddish/darker regions) correspond to the following properties: (i) a maximal level of nestedness, (ii) a small (sub-linear) mutualistic trade-off, and (iii) a high level of mutualistic strength within the constraint of any feasible solution being globally stable (49).

Importantly, a similar pattern is present in all the 23 observed mutualistic networks (45). For instance, using three different levels of interspecific competition $(\rho=0.2,0.4,0.6)$ we always find that structural stability is positively associated with nestedness and mutualistic strength (45). Similarly, structural stability is always associated with the mutualistic trade-off by a quadratic function, leading quite often to an optimal value for 
1 maximizing structural stability (45). These findings reveal that under the given charac-

terization of interspecific competition, there is a general pattern of network architecture that increases the structural stability of mutualistic systems.

$4 \quad$ Yet, one question remains to be answered: is the network architecture that we observe 5 in nature close to the maximum feasibility domain of parameter space under which species 6 coexist? To answer this question, we compare the observed network architecture with the7 oretical predictions. To extract the observed network architecture, we compute the observed nestedness from the observed binary interaction matrices (Table S1) following Ref. 42. The observed mutualistic trade-off $\delta$ is estimated from the observed number of visits of pollinators or fruits consumed by seed-dispersers to flowering plants $(41,50,51)$. The full details on how to compute the observed trade-off is provided in Note 52. Since there is no empirical data on the relationship between competition and mutualistic strength that could allow us to extract the observed mutualistic strength $\gamma_{0}$, our results on nestedness and mutualistic trade-off are calculated across different levels of mean mutualistic strength.

Figures 5B-D show that the observed network (blue solid lines) of the mutualistic system located in the grassland asclepiads of South Africa actually appears to have an architecture close to the one that maximizes the feasibility domain under which species coexist (reddish/darker region). To formally quantify the degree to which each observed network architecture is maximizing the set of conditions under which species coexist, we compare the net effect of the observed network architecture on structural stability against the maximum possible net effect. The maximum net effect is calculated in three steps.

First, as outlined in the previous section, we compute the partial fitted values of the effect of alternative network architectures on species persistence (48). Second, we extract the range of nestedness allowed by the network given the number of species and interactions in the system (45). Third, the maximum net effect of network architecture on structural stability is computed by finding the difference between the maximum and 
1 minimum partial fitted values within the allowed range of nestedness and mutualistic trade-off between $\delta \in[0, \ldots, 1.5]$. Note that all the observed mutualistic trade-offs have values between $\delta \in[0, \ldots, 1.5]$. Finally, the net effect of the observed network architecture on structural stability corresponds to the difference between the partial fitted values for the observed architecture and the minimum partial fitted values extracted in the third step described above.

Looking across different levels of mean mutualistic strength, Figure 6 shows that in the majority of cases (18 out of $23, P=0.004$, binomial test), the observed network architectures induce more than half the value of the maximum net effect on structural stability (red solid line). These findings reveal that observed network architectures tend to maximize the range of parameter space - structural stability - for species coexistence.

\section{Structural stability of systems with other interaction types}

In this section we explain how our structural stability framework can be applied to other types of interspecific interactions in complex ecological systems. We first explain how structural stability can be applied to competitive interactions. We proceed by discussing how this competitive approach can be used to study trophic interactions in food webs.

For a competition system with an arbitrary number of species, we can assume a standard set of dynamical equations given by $\frac{d N_{i}}{d t}=N_{i}\left(\alpha_{i}-\sum_{j} \beta_{i j} N_{j}\right)$, where $\alpha_{i}>0$ are the intrinsic growth rates, $\beta_{i j}>0$ are the competition interaction strengths, and $N_{i}$ is the abundance of species $i$. Recall that the Lyapunov-diagonal stability of the interaction matrix $\beta$ would imply the global stability of any feasible equilibrium point. However, in non-symmetric competition matrices, Lyapunov stability does not always imply Lyapunov-diagonal stability (53). This establishes that we should work with a restricted class of competition matrices such as the ones derived from the niche space of Ref. 54 . Indeed, it has been demonstrated that this class of competition matrices are Lyapunov- 
diagonally stable, and this stability is independent on the number of species (55). For a competition system with a symmetric interaction-strength matrix, the structural vector is equal to its leading eigenvector. For other appropriate classes of matrices, we can compute the structural vectors in the same way as we did with the effective competition matrices of our mutualistic model, and numerically simulate the feasibility domain of the competition system. In general, following this approach, we can verify that the lower is the average interspecific competition, the larger is the feasibility domain, and in turn the higher is the structural stability of the competition system.

In the case of predator-prey interactions in food webs, so far there is no analytical work demonstrating the conditions for a Lyapunov-diagonally stable system and how this is linked to its Lyapunov stability. Moreover, the computation of the structural vector of an antagonistic system is not a straightforward task. However, we may have a first insight about how the network architecture of antagonistic systems modulates their structural stability by transforming a 2-trophic-level food web into a competition system among predators. Using this transformation, we are able to verify that the higher is the compartmentalization of a food web, the higher is its structural stability (results not shown). In conclusion, there is no universal rule to study the structural stability of complex ecological systems. Each type of interaction poses their own challenges as function of their specific population dynamics.

\section{Discussion}

Here, we have investigated the extent to which different network architectures of mutualistic systems can provide a wider range of conditions under which species coexist. Note that this research question is completely different from the question of which network architectures are aligned to a fixed set of conditions. Previous numerical analysis based on arbitrary parameterizations were indirectly asking the latter, and previous studies based on local stability were not rigorously verifying the actual coexistence of species. Of 
1 course, if there is a good empirical or scientific reason to use a specific parameterization, 2 then we should take advantage of this. However, as the set of conditions present in a community can be constantly changing based on stochasticity, adaptive mechanisms, or global environmental change, we believe that understanding which network architectures can increase the structural stability of a community becomes a relevant question. Indeed, this is a question much more aligned with the challenge of assessing the consequences of global environmental change - by definition directional and large - than the alternative framework of linear stability, focusing on the responses of a steady state to infinitesimally small perturbations.

We advocate structural stability as an integrative approach to provide a general assessment of the implications of network architecture across ecological systems. Interestingly, our findings show that many of the observed mutualistic network architectures tend to maximize the domain of parameter space under which species coexist. This means that in mutualistic systems, both a nested network architecture and a small mutualistic trade-off are one of the most favorable structures for community persistence. Our predictions could be tested experimentally by exploring whether communities with an observed network architecture that maximizes structural stability stand higher values of perturbation. Similarly, our results open up new questions such as what the reported associations between network architecture and structural stability tell us about the evolutionary processes and pressures occurring in ecological systems.

Although the framework of structural stability has not been as dominant in theoretical ecology as the concept of local stability, it has a long tradition in other fields of research (29). For example, it has been key in evolutionary developmental biology to articulate the view of evolution as the modification of a conserved developmental program (27, 28). Thus, some morphological structures are much more common than others because they are compatible with a wider range of developmental conditions. This provided a more mechanistic understanding of the generation of form and shape through evolution 
1 (56) than that provided by a historical, functionalist view. We believe ecology can also 2 benefit from this structuralist view. The analogous question here would assess whether 3 the invariance of network architecture across diverse environmental and biotic conditions 4 is due to the fact that it is the one increasing the likelihood of species coexistence in an 5 ever-changing world. 


\section{References and Notes}

1. R. M. May, Nature 238, 413 (1972).

2. A. I. Ives, S. R. Carpenter, Science 317, 58 (2007).

3. T. J. Case, An Illustrated Guide to Theoretical Ecology (Oxford University Press, 2000).

4. S. L. Pimm, Food Webs (The University of Chicago Press, 2002).

5. A. Roberts, Nature 251, 607 (1974).

6. U. Bastolla, et al., Nature 458, 1018 (2009).

7. T. Okuyama, J. N. Holland, Ecol. Letts. 11, 208 (2008).

8. E. Thébault, C. Fontaine, Science 329, 853 (2010).

9. S. Allesina, S. Tang, Nature 483, 205 (2012).

10. A. James, J. W. Pitchford, M. J. Plank, Nature 487, 227 (2012).

11. S. Saavedra, D. B. Stouffer, Nature 500, E1 (2013).

12. S. Suweis, F. Simini, J. R. Banavar, A. Maritan, Nature 500, 449 (2013).

13. A.-M. Neutel, J. A. P. Heesterbeek, P. C. de Ruiter, Science 296, 1120 (2002).

14. B. S. Goh, Am. Nat. 111, 135 (1977).

15. D. O. Logofet, Linear Algebra and its Applications 398, 75 (2005).

16. D. O. Logofet, Matrices and Graphs: Stability Problems in Mathematical Ecology (CRC Press, 1992).

17. Y. M. Svirezhev, D. O. Logofet, Stability of Biological Communities (Mir Publishers, 1982).

18. Any matrix $B$ is called Lyapunov stable if the real parts of all its eigenvalues are positive, meaning that a feasible equilibrium at which all species have the same abundance is at least locally stable. A matrix $B$ is called D-stable if $D B$ is a Lyapunov stable matrix for any strictly positive diagonal matrix $D$. D-stability is a stronger condition than Lyapunov stability in the sense that it grants the local stability of any feasible equilibrium (15). In 
addition, a matrix $B$ is called Lyapunov-diagonally stable if there exists a strictly positive diagonal matrix $D$ such that $D B+B^{t} D$ is a Lyapunov stable matrix. This notion of stability is even stronger than D-stability in the sense that it grants not only the local stability of any feasible equilibrium point, but also its global stability (14). Importantly, a Lyapunovdiagonally stable matrix has all its principal minors positive. Also, its stable equilibrium (which may be only partially feasible, i.e., some species may have an abundance of zero) is unique (57). This means that there is no alternative stable state for a given parameterization of intrinsic growth rates (55). We have chosen the convention of having a minus sign in front of the interaction-strength matrix $B$. This implies that $B$ has to be positive Lyapunov stable (i.e., the real parts of all eigenvalues have to be strictly positive) so that the equilibrium point of species abundance is locally stable. This same convention applies for $D$-stability and Lyapunov-diagonal stability.

19. J. H. Vandermeer, Science 188, 253 (1975).

20. T. J. Case, Proc. Natl. Acad. Sci. U.S.A. 87, 9610 (1990).

21. All simulations were performed by integrating the system of ordinary differential equations using the function ode45 of Matlab. Species are considered to have gone extinct when their abundance is lower than 100 times the machine precision. While we present the results of our simulations with a fixed value of $\rho=0.2$ and $h=0.1$, our results are robust to this choice as long as $\rho<1(45)$. Note that in our dynamical system, the units of the parameters are abundance for $N, 1 /$ time for $\alpha$ and $h$, and $1 /$ (time-biomass) for $\beta$ and $\gamma$. Abundance and time can be any unit (e.g., biomass and years) as long as they are constant for all species.

22. V. I. Arnold, Geometrical Methods in the Theory of Ordinary Differential Equations 2nd (Springer, 1988).

23. R. V. Solé, J. Valls, J. theor. Biol. 155, 87 (1992).

24. A. N. Kolmogorov, Giornale Istituto Ital. Attuari 7, 74 (1936).

25. Y. A. Kuznetsov, Elements of Applied Bifurcation Theory (third edition) (Springer, 2004).

26. J. H. Vandermeer, Am. Nat. 104, 73 (1970).

27. P. Alberch, E. A. Gale, Evolution 39, 8 (1985). 
28. P. Alberch, Geobios 12, 21 (1989).

29. R. Thom, Structural Stability and Morphogenesis (Addison-Wesley Pub., 1994).

30. U. Bastolla, M. L assig, S. C. Manrubia, A. Valleriani, J. Theor. Biol. 235, 521 (2005).

31. J. Bascompte, Science 325, 416 (2009).

32. J. Bascompte, P. Jordano, C. J. Melián, J. M. Olesen, Proc. Natl. Acad. Sci. U.S.A. 100, $9383(2003)$.

33. J. N. Thompson, The Geographic Mosaic of Coevolution (University of Chicago Press, Chicago, 2005).

34. E. L. Rezende, J. E. Lavabre, P. R. Guimarães, P. Jordano, J. Bascompte, Nature 448, 925 (2007).

35. S. Saavedra, F. Reed-Tsochas, B. Uzzi, Nature 457, 463 (2009).

36. L. A. Burkle, J. C. Marlin, T. M. Knight, Science 339, 1611 (2013).

37. D. H. Wright, Am. Nat. 134, 664 (1989).

38. J. N. Holland, T. Okuyama, D. L. DeAngelis, Science 313, 1887b (2006).

39. S. Saavedra, R. P. Rohr, V. Dakos, J. Bascompte, Nature Comm. 4, 2350 (2013).

40. R. Margalef, Perspectives in Ecological Theory (Univ. Chicago Press, 1968).

41. D. P. Vázquez, et al., Oikos 116, 1120 (2007).

42. M. Almeida-Neto, P. Guimarães, P. R. Guimarães Jr, R. D. Loyola, W. Urlich, Oikos 117, 1227 (2008).

43. B. S. Goh, Am. Nat. 113, 261 (1979).

44. Y. Takeuchi, N. Adachi, H. Tokumaru, Mathematical Biosciences 42, 119 (1978).

45. Materials and Methods are available as supporting material on Science Online.

46. R. P. Rohr, R. E. Naisbit, C. Mazza, L.-F. Bersier, arXiv:1310.4633 (2013). 
47. P. McCullagh, J. A. Nelder, Generalized Linear Models (Chapter 4, 2nd edition) (Chapman and Hall, London, 1989).

48. For a given network, we look at the association of the fraction of surviving species with the deviation from the structural vector of plants $\eta_{(P)}$ and animals $\eta_{(A)}$, nestedness $N$, mutualistic trade-off $\delta$, and mean level of mutualistic strength $\bar{\gamma}=\left\langle\gamma_{i j}\right\rangle$ using the following binomial generalized linear model $(47)$ :

$\operatorname{logit}($ probability of surviving $) \sim \log \left(\eta_{A}\right)+\log \left(\eta_{P}\right)+\bar{\gamma}+\bar{\gamma}^{2}+\bar{\gamma} \cdot N+\bar{\gamma} \cdot N^{2}+\bar{\gamma} \cdot \delta+\bar{\gamma} \cdot \delta^{2}$

Obviously, at a level of mutualism of zero $(\gamma=0)$ nestedness and mutualistic trade-off cannot influence the probability of a species to survive. We have to include an interaction between the mean level of mutualism and the nestedness and mututalistic trade-off. We have also included a quadratic term in order to take into account potential non-linear effects of nestedness, mutualistic trade-off, and mean level of mutualistic strength. The effect of network architecture is confirmed by the significant likelihood ratio between the full model and a null model without such a network effect $(p<0.001)$ for all the observed empirical networks. The effect of network architecture on structural stability can be quantified by the partial fitted values defined as follows:

$$
\text { partial fitted values }=\hat{\beta}_{1} \bar{\gamma}+\hat{\beta}_{2} \bar{\gamma}^{2}+\hat{\beta}_{3} \bar{\gamma} \cdot N+\hat{\beta}_{4} \bar{\gamma} \cdot N^{2}+\hat{\beta}_{5} \bar{\gamma} \cdot \delta+\hat{\beta}_{6} \bar{\gamma} \cdot \delta^{2}
$$

where $\hat{\beta}_{1} \cdots \hat{\beta}_{6}$ are the fitted parameters corresponding to the terms $\bar{\gamma}$ to $\bar{\gamma} \cdot \delta^{2}$, respectively.

49. J. Bascompte, P. Jordano, J. M. Olesen, Science 312, 431 (2006).

50. P. Turchin, I. Hanski, Am. Nat. 149, 842 (1997).

51. P. Jordano, J. Bascompte, J. M. Olesen, Ecol. Letts. 6, 69 (2003).

52. To estimate the mutualistic trade-off, $\delta$, from an empirical point of view, we proceed as follows. First, there is available data on the frequency of interactions. Thus, $q_{i j}$ is the observed number of visits of animal species $j$ on plant species $i$. This quantity has been proven to be the best surrogate of per-capita effects of one species on another $\left(\gamma_{i j}^{P}\right.$ and $\left.\gamma_{i j}^{A}\right)(41)$. Second, the networks provide information on the number of species one species interacts with (its degree or generalization level; $k_{i}^{A}$ and $k_{i}^{P}$ ). Following Refs. 41,50,51, the generalization level of a species has been found to be proportional to its abundance at equilibrium. 
Thus, the division of the total number of visits by the product of the degree of plants and animals can be assumed to be proportional to the interaction strengths. Mathematically, we obtain two equations, one for the effect of the animals and the plants and vice versa: $\left(q_{i j}\right) /\left(k_{i}^{P} k_{j}^{A}\right) \propto \gamma_{i j}^{P}$ and $\left(q_{i j}\right) /\left(k_{i}^{A} k_{j}^{P}\right) \propto \gamma_{i j}^{A}$. Introducing the explicit dependence between interaction strength and trade-off, i.e., $\gamma_{i j}^{P}=\gamma_{0} /\left(k_{i}^{P}\right)^{\delta}$ and $\gamma_{i j}^{A}=\gamma_{0} /\left(k_{i}^{A}\right)^{\delta}$, we obtain $\left(q_{i j}\right) /\left(k_{i}^{P} k_{j}^{A}\right) \propto\left(\gamma_{0}\right) /\left(\left(k_{i}^{P}\right)^{\delta}\right)$ and $\left(q_{i j}\right) /\left(k_{i}^{A} k_{j}^{P}\right) \propto\left(\gamma_{0}\right) /\left(\left(k_{i}^{A}\right)^{\delta}\right)$. In order to estimate the value of $\delta$, we can just take the logarithm on both sides of the previous equations for the data excluding the zeros. Then $\delta$ is simply given by the slope of the following linear regressions:

$$
\log \left(\frac{q_{i j}}{k_{i}^{P} k_{j}^{A}}\right)=a^{P}-\delta \log \left(k_{i}^{P}\right) \quad \text { and } \quad \log \left(\frac{q_{i j}}{k_{i}^{A} k_{j}^{P}}\right)=a^{A}-\delta \log \left(k_{i}^{A}\right),
$$

where $a^{P}$ and $a^{A}$ are the intercept for plants and animals, respectively. These two regressions are performed simultaneously by lumping together the data set. Note that the intercept for the effect of animals on plants $\left(a^{P}\right)$ may not be the same as the intercept for the effect of the plants on the animals $\left(a^{A}\right)$.

53. The competition matrix given by $\beta=\left[\begin{array}{ccc}3 & 3.1 & 1 \\ 5 & 5 & 6 \\ 4 & 1 & 3\end{array}\right]$ is Lyapunov stable but not $D$-stable. As Lyapunov-diagonal stability implies $D$-stability, this matrix is also not Lyapunov-diagonally stable. This counter example was found by Steve Baigent (personal communication).

54. R. MacArthur, Theor. Pop. Biology 1, 1 (1970).

55. T. J. Case, R. Casten, Am. Nat. 113, 705 (1979).

56. D. W. Thompson, On Growth and Form (Cambridge Univ. Press, 1992).

57. Y. Takeuchi, N. Adachi, J. Math. Biol. 10, 401 (1980).

58. J. Ollerton, S. D. Johnson, L. Cranmer, S. Kellie., Ann. Botany 92, 807 (2003).

59. D. S. Watkins, Topics in Matrix Analysis (Definition 2.5.7) (Cambridge Univ. Press, 1991).

60. R. A. Horn, C. R. Johnson, Topics in Matrix Analysis (Cambridge Univ. Press, 1991).

61. C. R. Johnson, J. Econom. Theory 9, 53 (1974). 
We thank Ugo Bastolla, Louis-Félix Bersier, Vasilis Dakos, Antonio Ferrera, Miguel A. Fortuna, Luis J. Gilarranz, Sonia Kéfi, Jelle Lever, Bartolo Luque, Anje M. Neutel, Alberto Pascual-García, Daniel B. Stouffer, and Jason Tylianakis for insightful discussions. Funding was provided by the European Research Council through an Advanced Grant (JB) and FP7-REGPOT-2010-1 program under project 264125 EcoGenes (RPR). The data are publicly available at www.web-of-life.es.

\section{Supporting Online Material}

www.sciencemag.org/xxx

Figures S1-S14

Table S1

Methods 


\section{Figure captions}

- Fig. 1. Stability and feasibility of a two-species competition system. For the same parameters of competition strength (which grant the global stability of any feasible equilibrium), panels $\mathbf{A}, \mathbf{B}$, and $\mathbf{C}$ represent the two isoclines of the system. Their intersection gives the equilibrium point of the system $(3,19)$. Scenario A leads to a feasible equilibrium (both species have positive abundances at equilibrium), while in scenarios $\mathbf{B}$ and $\mathbf{C}$ the equilibrium is not feasible (one species has a negative abundance at equilibrium). Panel $\mathbf{D}$ represents the area of feasibility in the the parameter space of intrinsic growth rates, under the condition of global stability. This means that when the intrinsic growth rates of species are chosen within the white area, the equilibrium point is globally stable and feasible. In contrast, when the intrinsic growth rates of species are chosen within the green area, the equilibrium point is not feasible. Points A-C indicate the parameter values corresponding

- Fig. 2. Numerical analysis of species persistence as a function of model parameterization. This figure shows the simulated dynamics of species abundance and the fraction of surviving species (positive abundance at the end of the simulation) using the mutualistic model of Ref. 6. Simulations are performed using an empirical network located in Hickling Norfolk, UK (see Table S1), a randomized version of this network using the probabilistic model of Ref. 32, and the network without mutualism (only competition). Each row corresponds to a different set of growth rate values. Note that it is always possible to choose the intrinsic growth rates such that all species are persistent in each of the three scenarios and, at the same time, the community persistence defined as the fraction of surviving species is lower in the alternative scenarios.

- Fig. 3. Structural stability in a two-species competition system. The figure shows 
how the range of intrinsic growth rates leading to the stable coexistence of the two species (white region) changes as a function of the competition strength. Decreasing interspecific competition, from panel $\mathbf{A}$ to $\mathbf{D}$, increases the area of feasibility, and in turn the structural stability of the system. Here, $\beta_{11}=\beta_{22}=1$, and $\beta_{12}=\beta_{21}=\rho$. Our goal is extending this analysis to realistic networks of species interactions.

- Fig. 4. Deviation from the structural vector and community persistence. On panel A, we show the structural vector of intrinsic growth rates (in red) for the two-species competition system of Fig. 1. The structural vector is the vector in the center of the domain leading to the feasibility of the equilibrium point (white region), and thus can tolerate the largest deviation before any of the species goes extinct. The deviation between the structural vector and any other vector (in blue) is quantified by the angle between them. On panel $\mathbf{B}$, we show the effect of the deviation from the structural vector on intrinsic growth rates on community persistence defined as the fraction of model-generated surviving species. The example corresponds to an observed network located in North Carolina, USA (see Table S1) with a mutualistic trade-off $\delta=0.5$, and a maximum level of mutualistic strength $\gamma_{0}=0.2402$. Blue symbols represent the community persistence and the surface represents the fit of a logistic regression $\left(R^{2}=0.88\right)$.

- Fig. 5. Structural stability in complex mutualistic systems. For an observed mutualistic system with 9 plants, 56 animals, and 103 mutualistic interactions located in the grassland asclepiads in South Africa (see Table S1) (58), (A) corresponds to the effect - colored by partial fitted residuals — of the combination of different architectural values (nestedness, mean mutualistic strength, and mutualistic trade-off) on the domain of structural stability. The reddish/darker the color, the larger the parameter space compatible with the stable coexistence of all species, and in turn the 
larger the domain of structural stability. (B), (C), and (D) correspond to different slices of (A). Slice (B) corresponds to a mean mutualistic strength of 0.21 , slice (C) corresponds to the observed mutualistic trade-off, and slice (D) corresponds to the observed nestedness. Solid lines correspond to the observed values of nestedness and mutualistic trade-offs.

- Fig. 6. Net effect of network architecture on structural stability. For each of the 23 observed networks (Table S1), we show how close is the observed feasibility domain (partial fitted residuals) as function of the network architecture to the theoretical maximal feasibility domain. The network architecture is given by the combination of nestedness and mutualistic trade-off (x-axis) across different values of mean mutualistic strength (y-axis). The solid red and dashed black lines correspond to the maximum net effect and observed net effect, respectively. In 18 out of 23 networks (indicated by the asterisk), the observed architecture exhibits more than half the value of the maximum net effect (gray regions). Note that the net effect of each network architecture is system-dependent and cannot be used to compare across networks. 